\title{
An Enhanced Thermoelectric Property of 1-D Silicon Atomic Wire: An Ab-Initio Study
}

\author{
H. Joshi ${ }^{1, *}$, D. P. Rai ${ }^{2}$, P. K. Patra ${ }^{3}$, K. C. Bhamu ${ }^{4}$, R. K. Thapa ${ }^{1}$ \\ ${ }^{1}$ Department of Physics, Mizoram University, Aizawl 796004, India \\ ${ }^{2}$ Department of Physics, Pachhunga University College, Aizawl 796001, India \\ ${ }^{3}$ Faculty in Centre for Science Education, NEHU Shillong, 794022, India \\ ${ }^{4}$ Department of Physics, Goa University, Taleigao Plateau-403260, Goa, India
}

Copyright $\bigcirc 2016$ by authors, all rights reserved. Authors agree that this article remains permanently open access under the terms of the Creative Commons Attribution License 4.0 International License

\begin{abstract}
The band structure calculation was performed using the Full Potential Linearized Augmented Plane Wave Method (FP-LAPW) within a frame work of Density Functional Theory (DFT). We have observed a band gap in cubic bulk Silicon where as a Silicon atomic chain shows a Dirac cone like features along the $\Delta$-symmetry, $\sim 3.0 \mathrm{eV}$ above Fermi energy (EF). An indirect band gap of about $1.16 \mathrm{eV}$ is observed in case of bulk Si crystal. The narrow band gap has been shifted in case of Si-atomic wire to the conduction region. The shifted band gap in Si-nano structure indicates the semi-metal type behaviour, thereby increasing its electrical conductivity. The Boltzmann semi-classical transport theory is used to calculate the thermoelectric properties. The ZT value of Si atomic chain is $\sim 1.55$ at $120 \mathrm{~K}$, which is higher than the experimental value, 1.2.
\end{abstract}

Keywords DFT, GGA, Si Atomic Wire, Band Structures, Thermoelectric Properties

\section{Introduction}

Silicon nanowires and nanotubes are now considered to be the potential candidates for futuristic technology due to their low dimension, lead to quantum confinement effect [1]. This will develop a serious interest towards the making of nanostructure materials for nanoscale components of field-effect transistors [2], high sensitivity chemical for biological detectors [3] and optoelectronic devices [4]. The understanding of their structure, stability, and electron transport properties is a key. However, decreasing the size of nanowires at lower size is limited to a single molecular atomic-chain [5]. The recent advancement of technology enables the experimentalist to generate the nanowires, nanotubes, and atomic wires by means of scanning tunnelling microscope (STM), mechanically controllable break junction techniques, or transmission electron microscope [6-8]. After that, numerous theoretical and experimental studies are performed on the atomic wires. In a recent example, the spinning zigzag shape of monatomic gold wires was predicted which explained the current transmission electron microscopy (TEM) results [8]. A linear monoatomic nanowires, dimer and bulk of $\mathrm{Cu}, \mathrm{Ag}, \mathrm{Au}, \mathrm{Ni}$, $\mathrm{Pd}$ and $\mathrm{Pt}$ were investigated from first principles among all $\mathrm{Pt}$ and Au shows most stable monatomic structures [9]. Studer et al. have studied the atomic scale structural and electronic properties of ion implanted silicon samples using cross-sectional scanning tunneling microscopy [10]. A theoretical study of an ultra-stable 1D infinite monatomic mixed valent gold wire gives an excellent electronic property [11]. From density functional theory (DFT), it has been predicted that $\mathrm{Si}$ atomic wire undergoes two structural rearrangements when it undergoes compression zig-zag and translation [12]. The Si wires and arrays are metallic in nature reported from a tight-binding theory as well as from first principles [13-14]. Szczęśniak et al. reported the quantum electronic conductance across nanojunctions made of silicon-doped carbon wires between carbon leads [15]. Molecular dynamics simulations provide insight into the understanding of the dynamic structural evolution of the low-dimensional silicon materials, which has potential applications in the design and fabrication of the silicon-based nanodevices [16]. A nano-mechanical theory predicts $\mathrm{Si}$ nanowires with $<001>$ face which is a most stable structure [17]. The study of an equilibrium conductance of $\mathrm{Si}$ and $\mathrm{C}$ atomic wires placed between the $\mathrm{Al}$ (100) electrodes reported an oscillatory behavior at mediocre length [18]. Other than aforementioned properties, owing to its excellent bio-compatibility of Si-wire, it can also be used as an artificial nerve fiber to repair damage tissues, bio-sensors and possesses some unique electrical, optical, magnetic and thermal properties. The progress in nanotechnology surge the realization of low dimensional 
thermoelectric devices based on 1D nanowires, thin films, and two-dimensional superlattices. One key interest is modeling of a nanostructure beyond the dimension of nano-wire (nano-scale) i.e up to atomic-wire (atomic-scale) and studying its physical properties. Si nanowires show promising thermoelectric properties with $\mathrm{ZT}$ value equals to 0.40 [19]. Using the experimental thermal conductivity and reducing the the thickness of nanowires the value of $\mathrm{ZT}$ can be enhanced upto $\sim 1$ with n-type carriers [20]. In recent years thermoelectric materials are of great interest because it plays an important role in energy conversion between heat and electricity. Automotive exhaust, industrial processes, etc. generate an enormous amount of waste heat [21]. The designing of materials that can convert waste heat into an electrical energy is a look for new innovation. For many years, the development of thermoelectric material for practical application remains a daunting task. Still, the improvement of thermoelectric efficiency is an open challenge for the material scientists. In 1990's a resurgence of interest deviate in the field of nano-structures to enhance the thermoelectric efficiency [22]. The ability of a material to convert heat into electricity is measured by a dimensionless figure of merit $Z T$, given by,

$$
Z t=\frac{S^{2} \sigma T}{\kappa_{e}+\kappa_{p}}
$$

where, $\sigma$ is the electrical conductivity, $S$ is the Seebeck coefficient, $\kappa_{e}$ is the electronic contribution to thermal conductivity and $\kappa_{p}$ is the phononic contribution to thermal conductivity. Low dimensional materials provide the possibility of improved thermoelectric performance due to the change in its band energy near the Fermi level, which in turn related to $S, \sigma$ and $\kappa_{p}$.

Recently, large improvements in $Z T$ have been reported in Silicon nano-structures $(Z T \sim 1)$, as compared to the bulk ( $Z T_{\text {bulk }} \sim 0.1$ ) [23]. Most of these improvements have been a result of suppressed phonon conduction $\left(k_{p}\right)$. As suggested by various studies, low dimensionality can be beneficial for increasing the power factor $\left(\mathrm{S}^{2} \sigma\right)$ of the material as well [24]. Since, the band structures and the Seebeck coefficient $(S)$ are inter-related, we are able to calculate other thermoelectric parameters like $\sigma, \kappa_{e} / \tau$ etc. In our calculation, we emphasize mostly on the electron transport neglecting the lattice contribution $\left(k_{p}\right)$. The modified equation of the $Z T$ is,

$$
Z t=\frac{S^{2} \sigma T}{\kappa_{e}}
$$

Using this equation, we have calculated the thermoelectric figure of merit of 1-D silicon atomic wire.

\section{Model \& Calculation Method}

The monatomic Si-nano wire was designed by the super cell method in which the position of $\mathrm{Si}$ atom is taken as $(0.5$,
$0.5,0.5)$ and a vacuum of $20 \AA$ is applied along the $\mathrm{x}$-axis and $y$-axis in order to discontinue the atomic wave function and to break the crystal symmetry along the $\mathrm{x}$ and $\mathrm{y}$ direction. As a result of this, the repetition of the Si-atom takes place only along one direction i.e. along the $\mathrm{z}$-axis. The structure thus obtained is 1D Si atomic chain (periodic linear chain of identical atoms) as shown in figure 1(a). The construction of $\mathrm{Si}$ atomic wire is similar to $\mathrm{Fe}$ atomic wire [25]. The lattice constant ( $\mathrm{a}$ and $\mathrm{b}$ ) of the Si-wire has been changed to $20 \AA$ and the lattice constant of $\mathrm{c}$ is taken as $1.92 \AA$ [26] which is equivalent to the bond length of $\mathrm{Si}-\mathrm{Si}$. The bulk-Si crystallizes in fcc structure with lattice constants, $a=b=c=$ $5.43 \AA$ [Figure 1(b)]. The calculation was performed with a computational code called WIEN2K based on DFT [27]. A k-mesh of $1 \times 1 \times 24$ and $24 \times 24 \times 24$ are taken for Si-wire and bulk, respectively.

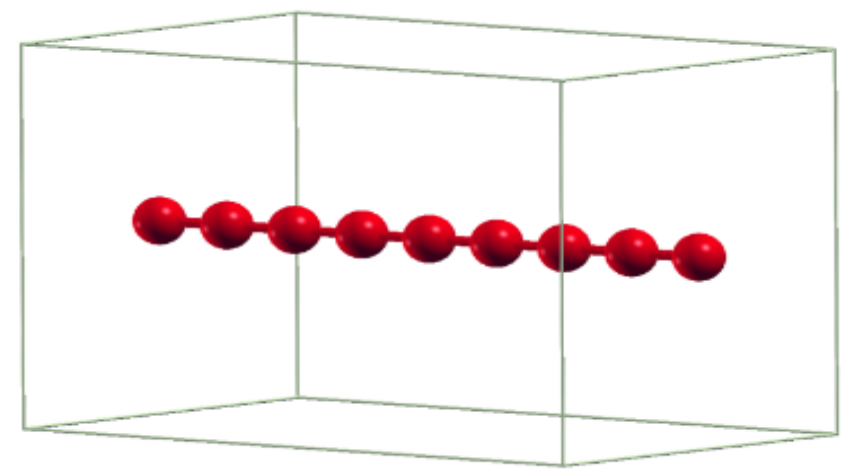

(a)

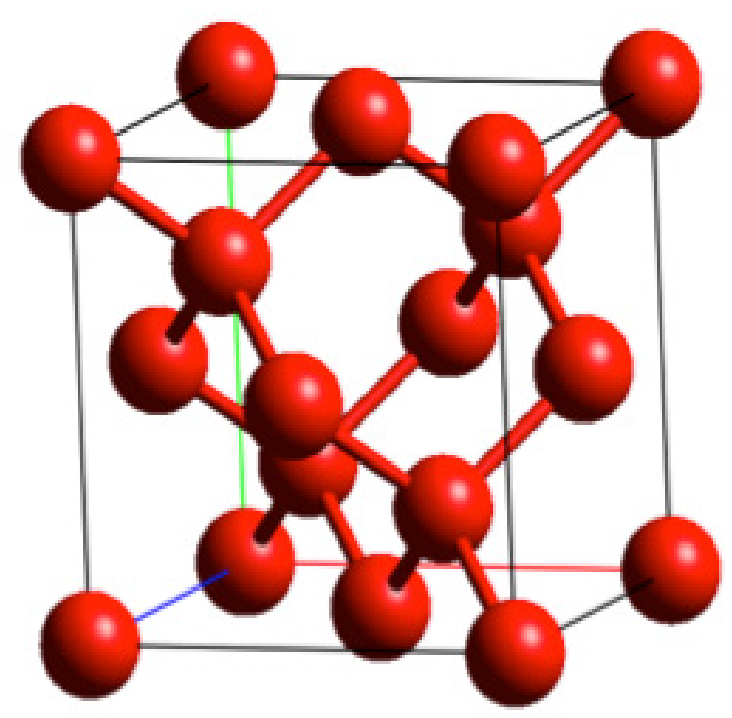

(b)

Figure 1. (a) 1-D silicon atomic chain; (b) FCC crystal structure of bulk silicon.

\section{Results}

We have calculated $\mathrm{S}, k_{e} / \tau$, and $Z T$ of 1-D silicon atomic 
wire as a function of temperature. At first, the energy band structure was calculated by using the FP-LAPW method. The band energy is used as an input in the BoltzTraP code for the calculation of thermoelectric thermoelectric properties [29].

\subsection{Energy Band Structure}

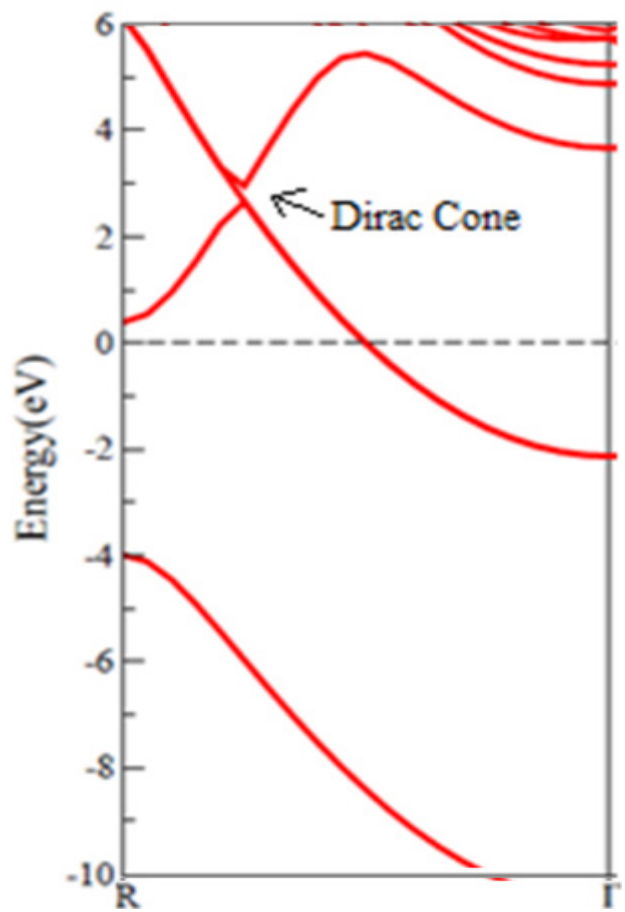

(a)

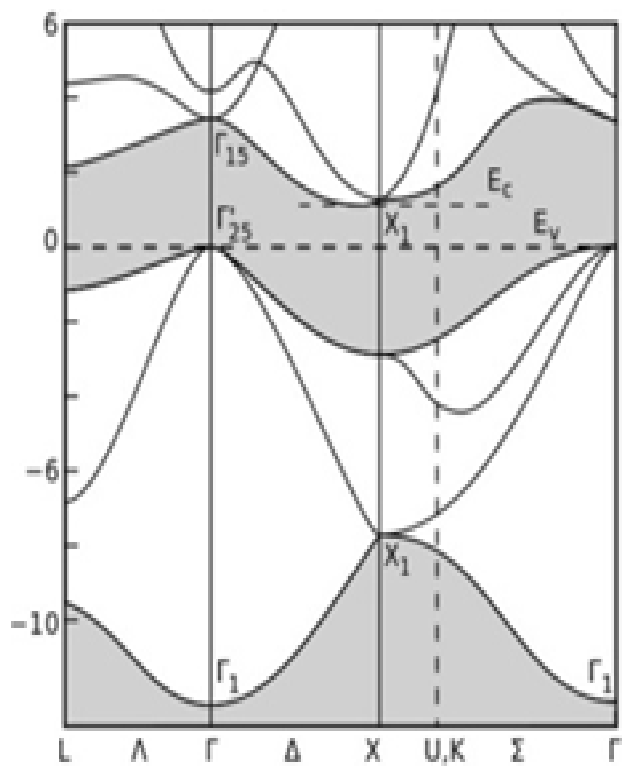

(b)

Figure 2. (a): band structure of 1-D silicon atomic and (b): band structure of bulk silicon crystal [28].

The band structure of 1-D silicon atomic wire is shown in figure 2(a). The most convenient feature of this system is that we can manipulate the Si-Si bond length to design the band structure and the resultant Fermi energy. We have observed a Dirac cone like features along the $\Delta$-symmetry, which lies above the Fermi energy. The formation of Dirac cone is due to electron effective mass. In our calculation, Dirac cone has been shifted by $\sim 3.0 \mathrm{eV}$ above the Fermi energy $\left(\mathrm{E}_{\mathrm{F}}\right)$. The shifting of Dirac cone towards the conduction band might due to the arbitrary choice of Si-Si bond length. The variation of $\mathrm{Si}-\mathrm{Si}$ bond length with minimum energy may set the Dirac cone exactly at the $\mathrm{E}_{\mathrm{F}}$. For comparison, we have taken the band structure of bulk $\mathrm{Si}$ crystal and it is shown in figure 2(b). The band structure plays an important role in electron transport. Previously reported that the modification in band structure of silicon nanowire effects in the electronic transport of field-effect-transistors (FET) which is used to evaluate the current-voltage characteristics $[2,29,30]$. The electronic property of high-index free-standing silicon nanowires with $<112>$ face of $0.8 \mathrm{~nm}$ is an indirect semiconductor; whereas $<110>$ wires have a direct band gap [17]. On the other hand, the limiting low dimension 1D-wires of $\mathrm{Si}$ diatomic molecules is metallic [13] which is in good agreement with our result. All the above calculated results predict that the the highest occupied level is $3 p$ which is partially filled in an isolated $\mathrm{Si}$ atom, and the Fermi energy is located in the dispersed $3 p$-band. This shows that it is not possible to obtain a finite band gap semiconductor with $\mathrm{Si}$ atoms having low dimensional atomic structures. At most, we can obtain the graphene like zero band gap by shifting the Dirac cone at the Fermi energy by tuning the Si-Si bond length. It is reported from previous study that the 1D-Si wires may exhibit an integer multiple of a quantum conductance in the linear response due to the transport of discrete momenta in the transverse directions and continuous momenta in the longitudinal direction [13].

An indirect band gap of about $1.16 \mathrm{eV}$ along $\Gamma-\mathrm{X}$ symmetry was reported in case of bulk Si crystal [8], where as in the case of $\mathrm{Si}$ atomic chain the cone like features are shifted entirely to the conduction region, showing almost narrow band gap. The shifting of narrow band gap in Si-wire indicates the existence of an intermediate state between metal and semiconductor, thereby increasing its electrical conductivity.

\subsection{Thermoelectric Properties}

The band structure is closely related to the Seebeck coefficient $(S)$ [see equation (3)], the electrical conductivity $(\sigma)$ and finally to its thermoelectric efficiency. The dense energy band near the Fermi level $\left(\mathrm{E}_{\mathrm{F}}\right)$ is responsible for high Seebeck coefficient and low value of electrical conductivity. The relation of Seebeck coefficient with the gradient of band energy near the Fermi level is shown in equation (3).

$$
S=\frac{\pi^{2}}{3} \frac{k_{B}^{2} T}{e}\left(\frac{d\{\ln [\sigma(E)]\}}{d E}\right)_{E=E_{F}}
$$



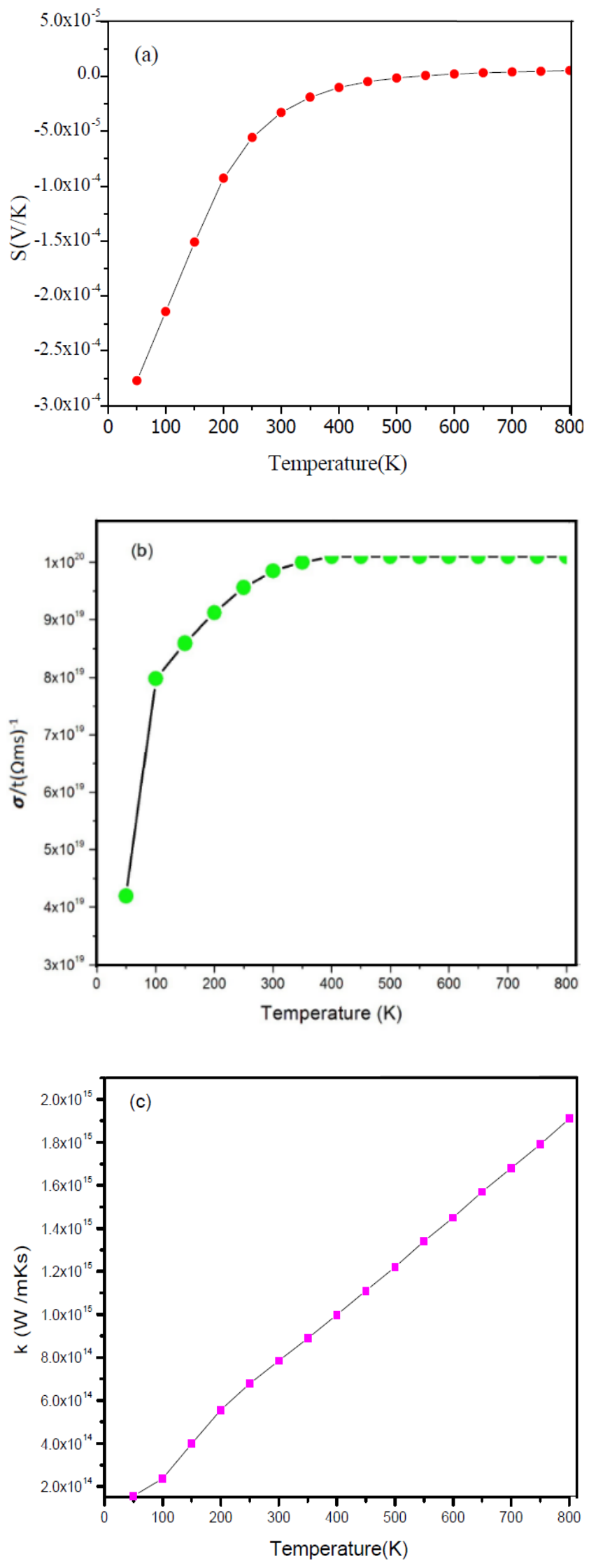

Figure 3. (a)Temperature dependence of Seebeck coefficient (S); (b) electrical conductivity over relaxation time $(\sigma / \tau)$; (c) electron thermal

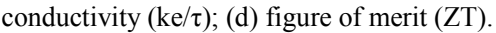

The value of calculated Seebeck coefficient(S) is plotted against temperature which is shown in Fig. 3(a). The maximum value of $S$ is $-2.77 \times 10^{-4} \mathrm{~V} / \mathrm{K}$ at $50 \mathrm{~K}$. Negative sign in $S$ indicates n-type carriers. The reason for low value of $S$ in our calculation is the absence of the dense bands near $\mathrm{E}_{\mathrm{F}}$ in the band structure (Fig. 3a). The plot of Seebeck coefficient against temperature shows linear behavior up to the room temperature and then the slope decreased, which is a typical behavior of low carrier density metal. The variation of an electrical conductivity divided by relaxation time against the temperature is shown in Fig. 3(b). From Fig. 3(b), Silicon atomic wire under study indicates semi-metallic behavior, as it gives a linear plot. The variation of thermal conductivity $\left(k_{e} / \tau\right)$ with temperature is shown in Fig. 3(c). The thermal conductivity shows linear behavior. The calculated value of $k_{e} / \tau$ is $1.91 \times 10^{15} \mathrm{~W} / \mathrm{mKs}$ at $800 \mathrm{~K}$. Figure 3 (d) gives the variation of figure of merit with temperature. The $Z T$ value calculated is 1.55 at $100 \mathrm{~K}$.

\section{Conclusions}

The formation of Dirac cone in case of Si-nano material indicates the presence of effective mass, which is absent in case of bulk materials. This implies that the band gap which was present in case of bulk material is being transformed into an overlapping in case of nano materials, which is the reason for increase in electronic conductivity. The $Z T$ value calculated is 1.55 at $100 \mathrm{~K}$, which is higher than the experimental value which is about 1.2. The reason for high ZT value 1.55 at $120 \mathrm{~K}$ might due to the absence of phonon contribution. Here in our calculation, the nano structure allows low scattering of phonons, as a result the lattice thermal conductivity is low. By some means, if we can reduce the mean free path of phonons to that length from which nano-structuring is effective, and then phononic contribution to thermal conductivity can be made minimum. 


\section{REFERENCES}

[1] Y. Cui, C. M. Lieber, Science, 291, 851 - 853 (2001)

[2] Y. Cui, Z. Zhong, D. Wang, W. U. Wang, C. M. Lieber, Nano Letters, 3, 149 - 152 (2003)

[3] R. Martel, V. Derycke, C. Lavoie, J. Appenzeller, K. K. Chan, J. Tersoff, P. Avouris, Phys. Rev. Lett., 87, 256805 (2001).

[4] Q. Kuang, C. Lao, Z. L. Wang, Z. Xie, and L. Zheng, J. Am. Chem. Soc., 129, 6070 - 6071 (2007)

[5] W. Lu and C. M. Lieber, Nature Mat. 6, 841 (2007)

[6] A. Dhirani, P.-H. Lin et al, J. Chem. Phys. 106, 5249 (1997).

[7] M. A. Reed et al, Science 278, 252 (1997).

[8] H. Ohnishi, Y. Kondo, and K. Takayanagi, Nature (London) 395, 780 (1998)

[9] E. Y. Zarechnaya, N. V. Skorodumova and S. I. Simak, Comput. Mat. Sc. 43, 522-530 (2008)

[10] P. Studer, S. R. Schofield, C. F. Hirjibehedin, and N. J. Curson, Appl. Phys. Lett. 102, 012107 (2013).

[11] C. Zhu, J. X. Liang and G. Wei Phys. Chem. Chem. Phys., 18, 12338-12343 (2016).

[12] S. Kausar and S. Joshi, Science and Technology, Special Issue: 32-40, (2013).

[13] T. Yamada, J. Vac. Sci. Technol. B 15, 1019 (1997).

[14] R. Rurali, Rev. Mod. Phys. 82, 427 (2010).

[15] D. Szczȩśniak, A. Khater, Z. Bąk, R. Szczȩśniak, and M. A. Ghantous, Nanoscale Res Lett. 7(1), 616 (2012).

[16] F. C. Wang, and Y. P. Zhao, Arch. Appl. Mech. 85, 323 (2015).

[17] H. Scheel, S. Reich and C. Thomsen, Phys. Stat. Sol. (b) 242, (2005).

[18] Y. H. Zhou et al. J. Phys.: Condens. Matter 20045225 (2008).

[19] E. B. Ramayya, L. N. Maurer, A. H. Davoody, and I. Knezevic, Phys. Rev. B 86, 115328 (2012).

[20] T. T. Vo, A. J.Williamson, V. Lordi, G. Galli, Nano Lett. 8(4):1111-4 (2008).

[21] A. Majumdar, Science, 303, 777-778 (2004).

[22] G. Chen, M.S. Dresselhaus, G. Dresselhaus, J.P. Fleurial and T. Caillat, Int. Mater. Rev. 48, 105-114 (2003).

[23] A. I. Hochbaum, R. Chen, R. D. Delgado, W. Liang, E. C. Garnett, M. Najarian, A. Majumdar, and P. Yang, Nature, 451, 163-167 (2008)

[24] L. D. Hicks and M. S. Dresselhaus, Phys. Rev. B 47,727-731 (1993).

[25] D. P. Rai, Sandeep, A. Shankar, P. K. Patra and R. K. Thapa, Nanoscience and Nanoengineering, 4, 52 - 57 (2016).

[26] A. Jepchcoat, H. Mao and P. Bell, Jour. Geophys. Research 91, 4677 - 4686 (1986).

[27] P. Blaha, G.K.H. Madsen, D. Kvasnicka, J. Luitz, WIEN2K, an augmented plane wave plus local orbitals program for calculating crystal properties (Vienna, Austria) 2008.

[28] G. K. H. Madsen and D.J. Singh, Comput. Phys. Commun. 175, 67-71 (2006).

[29] R. J. Chelikowsky, and M. L. Cohen, Phys. Rev. B10, 5095-5107 (1974).

[30] S. M. Koo, A. Fujiwara, J. P. Han, E. M. Vogel, C. A. Richter and J. E. Bonevich, Nano Lett. 4, 2197 (2004). 